\title{
Is It Contralateral Axillary Metastasis or Occult Breast Cancer?: A Confusing Case Report
}

\author{
Yong Gi Son Woon Won Kim Ki Hoon Kim Jin Soo Kim \\ Department of Surgery, Haeundae Paik Hospital, Inje University College of Medicine, \\ Busan, Korea
}

\section{Key Words}

Breast carcinoma - Occult primary tumor - Contralateral axillary lymph node metastasis . Immunohistochemistry

\begin{abstract}
We report the case of a 43-year-old woman with primary left breast cancer presenting metastatic lymphadenopathy in the contralateral axilla. This patient represents a diagnostic and therapeutic challenge because primary breast cancer, occult contralateral breast cancer, and extra-mammary primary lesion can all be the source of the contralateral axillary metastasis. Left breast-conserving surgery, left sentinel lymph node biopsy, right breast mass excision, and right axillary lymph node dissection were performed. Immunohistochemical analysis revealed that the left breast cancer specimen was positive for estrogen receptor (ER) and progesterone receptor (PR), but negative for human epidermal growth factor receptor 2 (HER2). In contrast, the right axillary lymphadenopathy specimen was negative for ER and PR, but positive for HER2. Further investigation revealed no evidence of occult primary cancers or extra-mammary tumors. After surgical intervention, the patient was treated with adjuvant chemotherapy, adjuvant radiation therapy, and targeted therapy with trastuzumab. Two years after diagnosis, she is free of disease and presently being treated with tamoxifen.
\end{abstract}

Published by S. Karger AG, Basel

\section{Introduction}

Contralateral axillary lymph node metastases (CAMs) are an uncommon finding in breast cancer. The reported incidence of CAM varies between 3.5 and $6 \%$ for all breast can-

KARGER125:s $\quad \begin{aligned} & \text { Dr. Woon Won Kim } \\ & \text { Department of Surgery, Haeundae Paik Hospital } \\ & \text { Inje University College of Medicine } \\ & \text { 1435, Jwa-dong, Haeundae-gu, Busan 612-030 (Korea) } \\ & \text { E-Mail hoopsper@hanmail.net }\end{aligned}$


Son et al.: Is It Contralateral Axillary Metastasis or Occult Breast Cancer?: A Confusing Case Report

cers [1-3]. Axillary lymph node metastasis from an occult breast cancer is also an uncommon presentation, accounting for $0.3-1 \%$ of all breast cancers. Traditionally, CAMs have been considered as stage IV disease. There are no standard guidelines for the management of CAMs at present. Moreover, clinicians may face management dilemmas in cases of CAM because they must consider the possibility of occult primaries in the contralateral breast or tumors outside the breast.

In most cases, CAMs and their primary tumors have similar histopathological features. Contrary to these expectations, we encountered a case in which synchronous contralateral metastatic lymphadenopathy and the primary breast cancer differed in terms of both hormonal receptor statuses and human epidermal growth factor receptor 2 (HER2) expression.

\section{Case Report}

A 43-year-old premenopausal woman presented with three non-palpable breast tumors on screening with breast ultrasonography. The breast tumor sizes were as follows: $6 \mathrm{~mm}$ [breast imaging-reporting and data system (BI-RADS) category 3] in the right breast, $6 \mathrm{~mm}$ (BI-RADS category 5) in the left breast, and $5 \mathrm{~mm}$ (BI-RADS category 3) in the left breast. Multiple pathological lymph nodes were discovered in the right axilla, but no pathological entities were found in the left axilla after ultrasonography.

Ultrasound-guided core needle biopsy was performed for the BI-RADS category 5 lesion in the left breast. The pathological diagnosis was invasive ductal carcinoma (IDC). Lesions of $8 \mathrm{~mm}$ (BI-RADS category 3) in the right breast, $4 \mathrm{~mm}$ (BI-RADS category 6) in the left breast, and $5 \mathrm{~mm}$ (BI-RADS category 3 ) in the left breast were found by magnetic resonance imaging. 18-Fluorodeoxyglucose positron emission tomography showed a positive uptake in the right axilla without abnormal hypermetabolic lesions in the breasts, and no definitive evidence of distant metastasis (fig. 1).

Left breast-conserving surgery, left sentinel lymph node biopsy, right breast mass excision, and right axillary lymph node dissection were performed. Histopathological examination of the left breast mass revealed a $0.7-\mathrm{cm}$ grade II IDC and no lymphovascular invasion. Histopathological investigation of the right breast mass indicated fibrocystic change. Although no metastasis was found in three left sentinel lymph nodes, three metastases were found in 25 right axillary lymph nodes (fig. 2a). On immunohistochemical analysis, the left breast tumor specimen was revealed to be positive for estrogen receptor (ER) (fig. 2b) and progesterone receptor (PR) (fig. 2c), but negative for HER2 (fig. 2d). In contrast, the right axillary lymphadenopathy specimen was negative for ER (fig. 2e) and PR (fig. 2f), but positive for HER2 (fig. 2g). Immunohistochemical analysis revealed positive cytokeratin 7 (CK7), negative CK20, positive CK19 (fig. 2h), and negative thyroid transcription factor-1 (TTF-1) staining in the right axillary lymphadenopathy. To exclude the possibility that the patient had other primary tumors, computed tomography of the neck, chest, and abdomen as well as gastrofiberscopy and colonofiberscopy were performed. These studies did not reveal any abnormal findings.

Postoperatively, the patient was treated with four cycles of adjuvant chemotherapy consisting of doxorubicin and cyclophosphamide as well as four cycles of docetaxel. After adjuvant chemotherapy, radiation therapy was administered to both breasts and right supraclavicular lymph nodes. Subsequently, she received targeted therapy with trastuzumab. Two years after diagnosis, she is free of disease and presently being treated with tamoxifen. 
Son et al.: Is It Contralateral Axillary Metastasis or Occult Breast Cancer?: A Confusing Case Report

\section{Discussion}

Lymph node metastasis of breast cancer mostly occurs in the ipsilateral axilla. In most studies of CAM, metachronous CAMs account for the majority of cases. In a case series by Devitt and Michalchuk [2], only 2 of 52 patients had synchronous CAM. Further, in a case series by Huston et al. [4], only 1 of 7 patients had synchronous CAM.

Morcos et al. [5] reported aggressive histopathological features in patients who have breast cancer with CAM, such as higher tumor grade, lymphovascular invasion, larger primary tumors, ER-negativity, and HER2 overexpression. However, in our report, the primary breast tumor was a grade II IDC, with ER-positivity, no lymphovascular invasion, and no HER2 overexpression.

As noted previously, the histopathological and immunohistochemical features of CAM are generally similar to those of the primary breast cancer. For example, Huston et al. [4] observed the same histological and immunohistochemical features in CAMs and their primary tumors in the contralateral breasts. However, in our case, immunohistochemical analysis of the primary breast lesion revealed ER+, PR+, and HER2- tissue, even though the contralateral axillary lymphadenopathy was ER-, PR-, and HER2+. These findings can make it difficult for clinicians to predict disease prognosis and establish a therapeutic strategy. If an occult breast cancer is a source of the metastatic lymphadenopathy in the contralateral axilla, the present case might be classified as bilateral breast cancer.

Breast magnetic resonance imaging has been helpful to detect occult primary lesions in the breast [6, 7]. In our case, breast magnetic resonance imaging to detect the presence of an occult primary tumor found a benign mass (BI-RADS category 3 ) in the right breast. Histopathological examination of the mass revealed fibrocystic change. Although we undertook imaging studies and other investigations to identify extra-mammary tumors, no abnormal findings were uncovered.

CK7-positive, CK20-negative, CK19-positive, and TTF-1-negative stainings were found in the immunohistochemical studies of CAM. The combination of these findings suggests that the breast is the primary site of the axillary lymph node metastasis [8].

Contralateral lymphadenopathy with primary breast cancer may occur because of CAM, an occult primary tumor in the contralateral breast, or an extra-mammary tumor. To confirm the diagnosis of synchronous CAM, it is essential to exclude the presence of occult primary cancers and extra-mammary tumors. This case suggests that specific tests and thorough investigations should be performed by clinicians who may face cases of dissimilar CAM and primary breast cancer. We have reported this case because it presents initial evidence regarding the very rare occurrence of synchronous CAM and primary breast cancer with different histopathological features.

\section{Statement of Ethics}

The patient provided written informed consent for her inclusion in this case report. Further, the case report was approved by our institution's ethics committee..

\section{Disclosure Statement}

The authors declare that they have no conflicts of interest. 


\section{Case Reports in Oncology}

\begin{tabular}{l|l}
\hline Case Rep Oncol 2015;8:498-502 \\
\hline DOI: $10.1159 / 000440662$ & $\begin{array}{l}\text { (c) 2015 The Author(s). Published by S. Karger AG, Basel } \\
\text { www.karger.com/cro }\end{array}$ \\
\hline
\end{tabular}

Son et al.: Is It Contralateral Axillary Metastasis or Occult Breast Cancer?: A Confusing Case Report

\section{References}

1 Daoud J, Meziou M, Kharrat M, et al: Contralateral axillary lymph node metastasis of cancer of the breast (in French). Bull Cancer 1998;85:713-715.

2 Devitt JE, Michalchuk AW: Significance of contralateral axillary metastases in carcinoma of the breast. Can J Surg 1969;12:178-180.

-3 Lanitis S, Behranwala KA, Al-Mufti R, Hadjiminas D: Axillary metastatic disease as presentation of occult or contralateral breast cancer. Breast 2009;18:225-227.

4 Huston TL, Pressman PI, Moore A, et al: The presentation of contralateral axillary lymph node metastases from breast carcinoma: a clinical management dilemma. Breast J 2007;13:158-164.

5 Morcos B, Jaradat I, El-Ghanem M: Characteristics and therapeutic options for contralateral axillary lymph node metastasis in breast cancer. Eur J Surg Oncol 2011;37:418-421.

6 Morris EA, Schwartz LH, Dershaw DD, et al: MR imaging of the breast in patients with occult primary breast carcinoma. Radiology 1997;205:437-440.

7 Orel SG, Weinstein SP, Schnall MD, et al: Breast MR imaging in patients with axillary node metastases and unknown primary malignancy. Radiology 1999;212:543-549.

8 National Comprehensive Cancer Network: Clinical Practice Guidelines in Oncology, Occult Primary. Version 3.2014. http://www.nccn.org/professionals/physician_gls/f_guidelines.asp\#occult (accessed November 30, 2014).

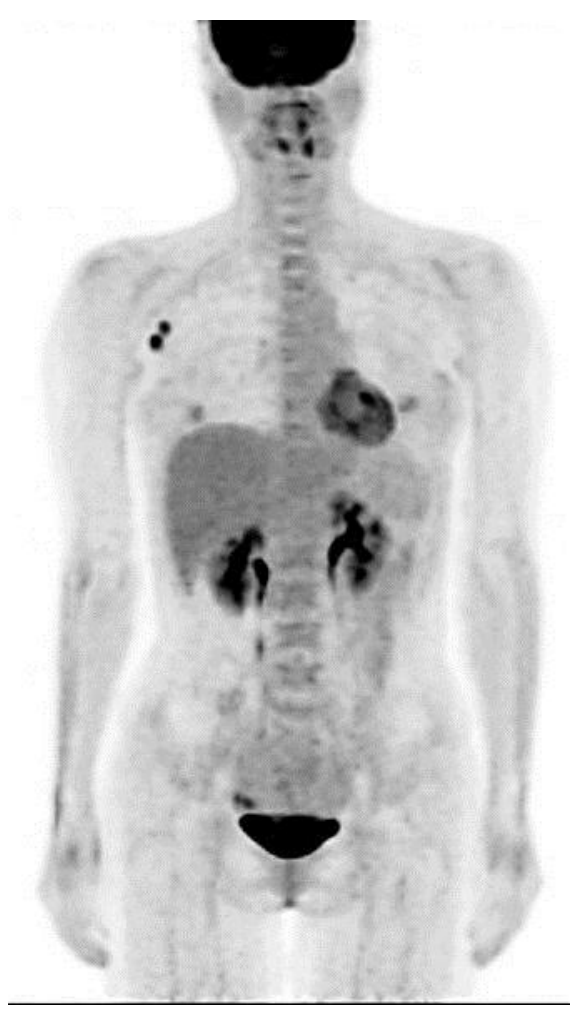

Fig. 1. Preoperative positron emission tomography scan. No definite abnormal hypermetabolic lesion was observed in either breast. An abnormal uptake of fluorodeoxyglucose was observed in the right axillar level I. 


\section{Case Reports in Oncology}

\begin{tabular}{l|l}
\hline Case Rep Oncol 2015;8:498-502 \\
\hline DOI: 10.1159/000440662 & $\begin{array}{l}\text { C 2015 The Author(s). Published by S. Karger AG, Basel } \\
\text { www.karger.com/cro }\end{array}$ \\
\hline
\end{tabular}

Son et al.: Is It Contralateral Axillary Metastasis or Occult Breast Cancer?: A Confusing Case Report
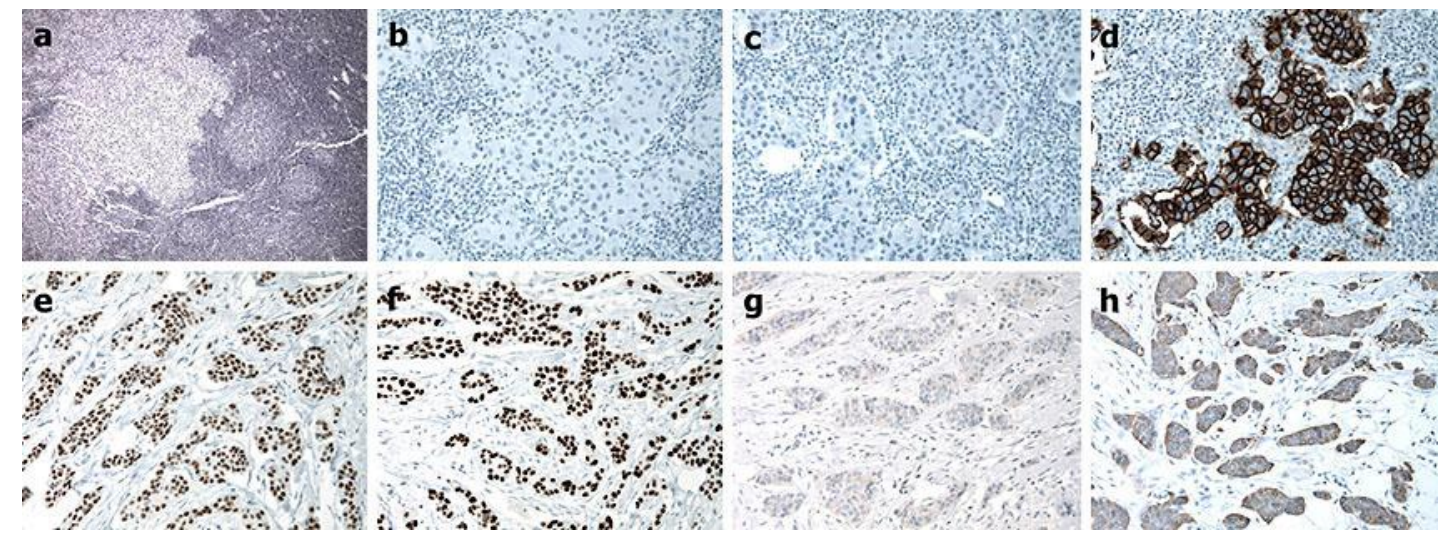

Fig. 2. Metastatic carcinoma cells from the right axillary lymphadenopathy $(\mathbf{a}-\mathbf{d}, \mathbf{h})$. Low power view of hematoxylin and eosin staining (a). There was no ER (b) or PR (c) staining. HER2 staining showed a strong complete membranous pattern (d). Infiltrating ductal carcinoma cells from the left breast (e-g). ER (e) and PR (f) showed strong nuclear expression in most of the tumor cells. HER2 did not show any membranous staining (g). Metastatic carcinoma cells from the right axillary lymphadenopathy were positive for CK19 (h). Original magnification $\times 100$ in a; Original magnification $\times 400$ in $\mathbf{b}-\mathbf{h}$. 